\title{
riccafd
}

Revista Iberoamericana de Ciencias de la Actividad Física y el Deporte

\section{EFECTOS DE UN PROGRAMA DE EJERCICIO INTRADIÁLISIS EN PACIENTES CON ENFERMEDAD RENAL CRÓNICA}

\section{EFFECTS OF AN INTRADIALYSIS EXERCISE PROGRAM IN PATIENTS WITH CHRONIC KIDNEY DISEASE}

Vázquez Rigueira, P1; Pedrero Chamizo, R ${ }^{2}$; Aparicio Ugarriza, R ${ }^{2}$; Santiago Dorrego C' $\mathrm{C}^{1}$; Calonge, S²; Gómez, F⿻; Palacios Le Ble ${ }^{2}$, G; Díaz Manjón, $\mathbf{R}^{3}$; Meléndez, A²; Barrios, L4; Botella, A3; González Gross, M²; Pérez Ruiz, M¹

'Vázquez Rigueira, P. Escuela de Doctorado e Investigación. Universidad Europea de Madrid. patricia.vazquez.rigueira@gmail.com

${ }^{2}$ Pedrero Chamizo, R. Grupo de investigación ImFINE. Universidad Politécnica de Madrid. raquel.pedrero@upm.es

${ }^{2}$ Aparicio Ugarriza, R. Grupo de investigación ImFINE. Universidad Politécnica de Madrid. apariciougarriza.raquel@gmail.com

'Santiago Dorrego, C. Grupo de investigación EsBIDA. Facultad de ciencias de la actividad física, deporte y fisioterapia Universidad Europea de Madrid.

catalina.santiago@universidadeuropea.es

${ }^{2}$ Calonge, S. Grupo de investigación ImFINE. Universidad Politécnica de Madrid.

sergio.calonge.pascual@gmail.com

${ }^{1}$ Gómez, F. Facultad de ciencias de la salud Univ. Int. de La Rioja. felix.gomez@unir.net

${ }^{2}$ Palacios Le Ble, G. Grupo de investigación ImFINE. Universidad Politécnica de Madrid. gonzalopalacios88@gmail.com

${ }^{3}$ Díaz Manjón, R. Fundación Renal Íñigo Álvarez de Toledo. Madrid.

rakelwendy89@hotmail.com

${ }^{2}$ Meléndez, A. Grupo de investigación ImFINE. Universidad Politécnica de Madrid. agustin. melendez@upm.es

${ }^{4}$ Barrios, L. Centro Técnico de Informática. Consejo Superior de Investigaciones Científicas.

laura@cti.csic.es

${ }^{3}$ Botella, A. Fundación Renal Íñigo Álvarez de Toledo. Madrid. abotella@friat.es

${ }^{2}$ González Gross, M. Grupo de investigación ImFINE. Universidad Politécnica de Madrid. marcela.gonzalez.gross@upm.es

${ }^{1}$ Pérez Ruiz, M. Grupo de investigación EsBIDA. Facultad de ciencias de la actividad física, deporte y fisioterapia Universidad Europea de Madrid. margarita.perez@universidadeuropea.es

Agradecimientos: Premios Liberbank de investigación en Medicina del Deporte en la Escuela de Medicina de la Educación Física y del Deporte de la Universidad de Oviedo en su edición de 2016. Este estudio ha sido financiado por la Cátedra Real Madrid-Universidad Europea (2016RM/09) y por la Fundación Renal Íñigo Álvarez de Toledo (FRIAT) (P141115303).

Correspondencia: Vázquez Rigueira, P: patricia.vazquez.rigueira@gmail.com

Código UNESCO: 241106 Fisiología del Ejercicio

Clasificación Consejo de Europa: 17. Actividad Física y Salud

Recibido el 31 de octubre de 2019

Aceptado el 8 de diciembre de 2019

DOI: http://dx.doi.org/10.24310/ 10.24310/riccafd.2019.v8i3.7490 


\section{RESUMEN}

El objetivo de este estudio fue comprobar el grado de cumplimiento y analizar el efecto de un programa intradiálisis de ejercicio físico sobre la condición física y salud del paciente con enfermedad renal crónica (ERC) que recibe hemodiálisis. Estudio de intervención con dos periodos de 14 semanas, uno sin intervención de ejercicio y otro con intervención, combinando trabajo de resistencia aeróbica y fuerza, en sesiones de 60 minutos, 3 días/semana. La condición física se evaluó a través de la capacidad de fuerza máxima de prensión manual, fuerza resistencia en extremidades inferiores y capacidad cardiorrespiratoria. El $62 \%$ de los pacientes cumplieron el $75 \%$ del programa. Durante el periodo sin ejercicio intradiálisis se observó una disminución significativa de la condición física, que se frenó durante el periodo con intervención de ejercicio. El programa de ejercicio intradiálisis es importante para frenar la pérdida de condición física ocasionada por la ERC.

Palabras clave: enfermedad renal crónica, hemodiálisis, ejercicio, capacidad aeróbica, fuerza.

\section{ABSTRACT}

The aim of this study was to check the grade of enforcement and to assess the effect of an intradialytic exercise program on fitness and health in hemodialysis patients. The design of this study consisted of 2 periods of 14 weeks, the first one without exercise and the next one with exercise. The program included both aerobic endurance and strength exercise in sessions of 60 minutes, 3 days per week. Physical condition was evaluated through handgrip strength, lower extremity strength and aerobic capacity. $62 \%$ of patients completed $75 \%$ of the exercise program. A statistically significant decrease in all fitness parameters was observed in period without exercise. This decrease stopped, due to the intervention of exercise program, without significance improvement. Intradialytic exercise program is important to reduce the loss of fitness associated to chronic kidney disease.

Key words: chronic kidney disease, hemodialysis, exercise, aerobic capacity, strength.

\section{INTRODUCCIÓN}

La Enfermedad Renal Crónica (ERC), afecta a un 8-16\% de la población mundial ${ }^{1}$ y a un $5 \%$ de los españoles. Según el Documento Marco sobre ERC dentro de la Estrategia de Abordaje a la Cronicidad en el Sistema Nacional de Salud (SNS), aproximadamente 4 millones de personas padecen ERC en España y, de ellas, más de 50.000 están en Tratamiento Renal Sustitutivo (TRS). Además, se estima que cada año, unas 6.000 personas con insuficiencia renal progresan hasta la necesidad de someterse a uno de los tres tipos de TRS: hemodiálisis (HD), diálisis peritoneal (DP) y trasplante renal (Tx). 
Este incremento estimado es debido a que es una enfermedad de etiología muy variable. Así, destacan la hipertensión arterial (HTA) y la diabetes mellitus (DM), cuyas prevalencias son muy altas en la población mundial actual, y el envejecimiento de la población, que está ocasionando un notable incremento de los pacientes con nefropatía vascular y de las enfermedades ya referidas.

La ERC conlleva una afectación sistémica importante, destacando la pérdida de masa muscular del paciente ${ }^{2}$. Factores como la acidosis ${ }^{3}$, la malnutrición, las co-morbilidades, el uso de corticoides, la edad biológica, el estrés oxidativo y el tratamiento de diálisis ${ }^{4}$ contribuyen al estado catabólico, la atrofia muscular y la infiltración de grasa intramuscular². Por otra parte, el desuso muscular ocasionado por el comportamiento sedentario durante el tiempo de diálisis y fuera del mismo es de un $64 \%$ en esta población ${ }^{5}$. La intolerancia al ejercicio y la inactividad son características establecidas en el enfermo renal crónico bajo tratamiento de HD. Se calcula que sólo el tiempo medio que precisa el tratamiento de la diálisis, hace que el enfermo permanezca en actitud sedentaria hasta 580 horas al año ${ }^{6}$. Este comportamiento sedentario junto al estado catabólico propio de la enfermedad renal conlleva una pérdida de masa muscular con nefastas consecuencias orgánicas que producen una disminución de la fuerza muscular y de la función muscular, una disminución de la condición física general, de la supervivencia y pronóstico de la enfermedad y de la calidad de vida ${ }^{7}$.

El entrenamiento de fuerza progresivo y de alta intensidad ha sido utilizado para mejorar la función muscular en personas de edad y frágiles y en aquellas con enfermedades crónicas debilitantes ${ }^{8}$. La alta prevalencia de catabolismo muscular en ERC sugiere que este tipo de entrenamiento sería muy beneficioso para la evolución de su enfermedad. Así, los trabajos de Johansen et al, informan que los pacientes con ERC pueden conseguir mejoras del área muscular después de 12 semanas de ejercicio de fuerza de intensidad moderada ${ }^{9}$.

El propósito de estudio fue analizar el efecto de un programa intradiálisis de ejercicio sobre la condición física del enfermo renal crónico y evaluar el grado de cumplimiento del programa de ejercicio. Nuestra hipótesis de trabajo es que la intervención de ejercicio controlado durante la sesión de diálisis con un grado de cumplimiento superior al $75 \%$ y durante 14 semanas mejora la condición física en el enfermo renal crónico incidiendo en la mejora de las actividades de la vida diaria.

\section{MATERIAL Y METODOS}

\section{Sujetos:}

Los sujetos participantes en el estudio fueron pacientes de la Fundación Renal Íñigo Álvarez de Toledo (FRIAT). De los 235 pacientes elegibles para participar en el estudio, 54 sujetos accedieron a participar y, un total de 29 sujetos finalizaron el programa de intervención (ver Figura 1).

Los criterios de inclusión fueron: a) Varones con edad entre 55 y 85 años; b) No presentar en el momento actual enfermedad cardiovascular ni angina inestable; c) No presentar enfermedad vascular cerebral ni alteraciones locomotoras 
o respiratorias que no permitan hacer el programa de ejercicio; d) No presentar osteoporosis avanzada; d) Fracción de eyección >45\%, Hb>10 g/dl; e) Acceso vascular funcionante. Los criterios de exclusión fueron: a) Haber presentado infarto de miocardio en las 6 semanas previas; b) Angina inestable al ejercicio o en reposos; c) Enfermedad vascular cerebral reciente o con riesgo de recidiva; d) Alteraciones músculo-esqueléticas, respiratorias (EPOC con descompensaciones frecuentes), enfermedad vascular periférica, enfermedad hepática activa, osteoporosis diagnóstica avanzada, aquellos en los que a criterio médico esté contraindicado el ejercicio físico; e) Encontrarse en alguna de las siguientes circunstancias: fracción de eyección cardiaca $<45 \%, \mathrm{Hb}<10 \mathrm{~g} / \mathrm{dl}$, acceso vascular problemático, tanto fístulas inmaduras o con riesgo de extravasación como disfunciones del catéter de características postural.

Todos los pacientes participantes tomaban fármacos antihipertensivos inhibidores de la enzima de conversión de angiotensina y antagonistas de los receptores de angiotensina II (IECA-ARAII), dieta hipoproteica, restricción salina, calcitriol, quelantes del fósforo, hierro y factores eritropoyéticos adaptando la dosis para mantener $\mathrm{Hb}>10 \mathrm{~g} / \mathrm{dl}$, y algunos necesitaban estatinas, antiagregantes y antidiabéticos orales.

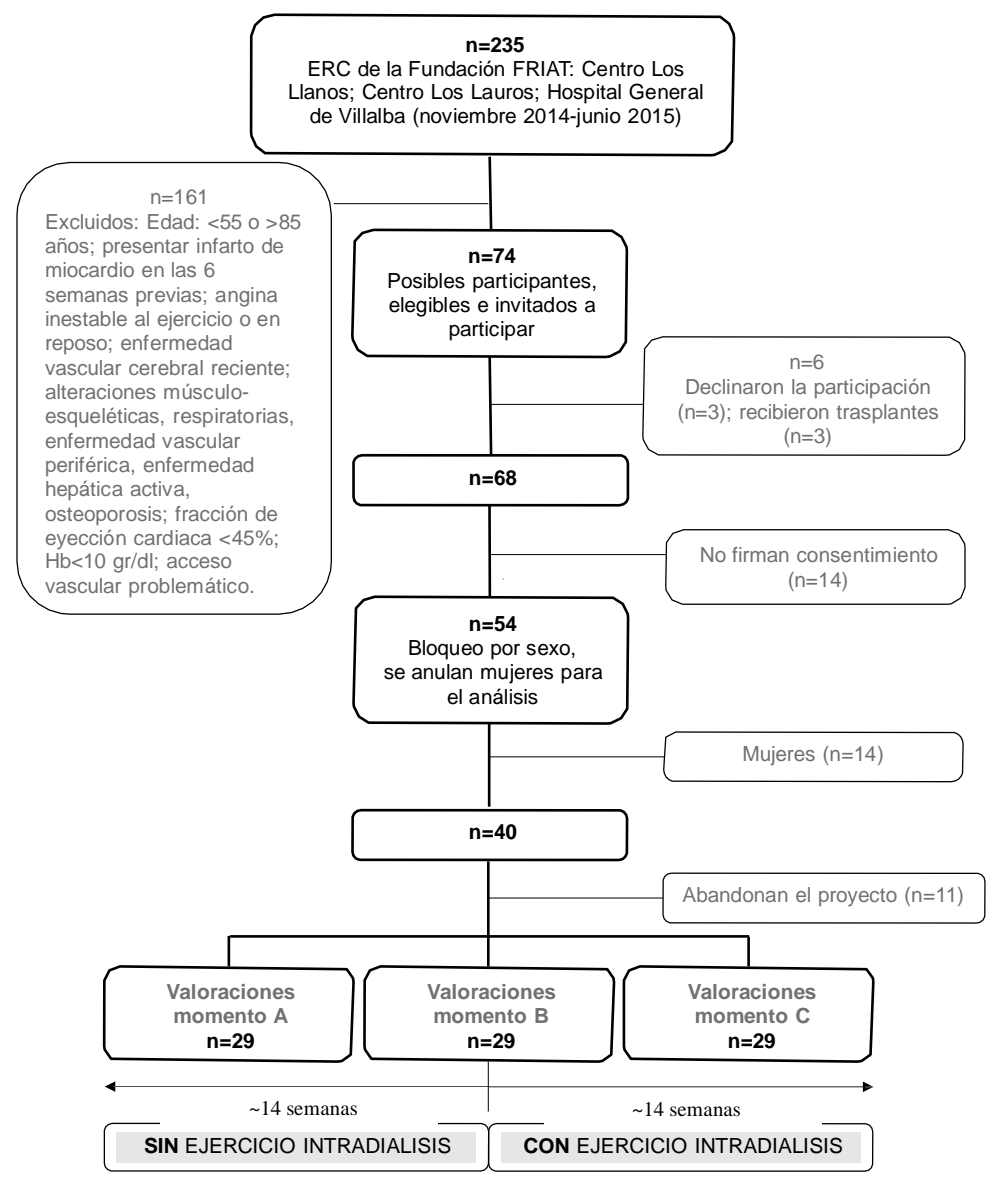

Figura 1. Diagrama de flujo de los pacientes con ERC sometidos a hemodiálisis participantes en el programa de ejercicio intradiálisis. 


\section{Diseño:}

Estudio de intervención en el que participaron 3 centros de la Fundación Renal Íñigo de Álvarez de Toledo (FRIAT) ubicados en la Comunidad de Madrid. Dada la heterogeneidad clínica de los participantes con ERC, se decidió que los propios sujetos fueran su grupo control, para lo cual se establecieron dos periodos de tiempo de 14 semanas, el primero sin intervención de ejercicio (periodo A-B) y el segundo con intervención de ejercicio (periodo B-C). Las valoraciones realizadas se llevaron a cabo en cada uno de los tres momentos señalados (momento $\mathrm{A}$, momento $\mathrm{B}$ y momento $\mathrm{C}$ ).

Este estudio fue aprobado por el Comité Ético de Investigación Clínica de la Fundación Universitaria Hospital de Alcorcón. Todos los pacientes firmaron un consentimiento informado de participación en el proyecto. El protocolo se ha realizado siguiendo las directrices de la Declaración de Helsinki y adendas posteriores.

\section{Valoración de la condición física:}

Todas las pruebas de evaluación fueron realizadas antes de la conexión al tratamiento de diálisis. Se valoraron la capacidad aeróbica y la fuerza muscular.

La valoración de la capacidad aeróbica en el paciente con ERC se midió a través del test " 6 minutes walk test" (6MWT) en los tres momentos A, B y C adaptado de la batería Senior Fitness Test (SFT) de Rikli y Jone ${ }^{10}$ durante el cual el sujeto debía desplazarse andando a la máxima velocidad para recorrer la mayor distancia durante 6 minutos de tiempo.

En cuanto a la fuerza muscular, se midió la fuerza máxima de prensión manual (DIN), en ambas manos, mediante un dinamómetro manual (modelo T.K.K. 5401, rango 5-100 kg, precisión 0,1 kg, fabricado en Japón).

Antes de proceder a la medición se explicó a cada uno de los sujetos el modo de realizar la prensión manual y se les instó para que realizaran un par de pruebas para familiarizarse con el gesto y el instrumental.

Para la medición, siguiendo el protocolo de la batería EUROFIT ${ }^{11}$, el sujeto se encontraba en bipedestación, si era posible, con las extremidades superiores a lo largo del cuerpo y en posición neutra de rotación. El agarre se realizaría de forma que las articulaciones interfalángicas proximales se encontrasen a $90^{\circ}$ para realizar la prensión y, a la hora de realizar el gesto, el paciente debería de evitar movimientos tales como flexiones y/o inclinaciones de muñeca y flexiones de codo. Se realizaron dos mediciones en cada una de las manos, con un minuto de descanso.

La fuerza-resistencia de las extremidades inferiores se analizó a través de la prueba funcional validada "30 and 60-second chair stand test" (STS30 y STS60), durante ambos tests el paciente debía levantarse y sentarse el máximo número de veces durante 30 o 60 segundos (12). También se valoró el test funcional de "sit-to-stand-to-sit" (STS10) en el que el sujeto debía de realizar una extensión completa de su cuerpo al levantarse de una silla y 
sentarse nuevamente hasta completar un total de 10 repeticiones en el menor tiempo posible ${ }^{12}$.

\section{Programa de intervención de ejercicio intradiálisis:}

El programa de entrenamiento intradiálisis estuvo adaptado a las características individuales de los sujetos y siempre cuidando la funcionalidad de la fístula.

La duración del programa fue de 14 semanas con una frecuencia de 3 días/ semana. El tipo de ejercicio combinado (fuerza y resistencia aérobica) y la duración de cada sesión fue de 60 minutos.

Cada sesión de ejercicio constaba de una parte inicial con ejercicios de movilidad articular y ejercicios respiratorios que ayudaban a provocar una adaptación cardiorrespiratoria y locomotora, y una parte principal de ejercicio de fuerza, donde la carga de entrenamiento se adaptó a la condición física de los pacientes semana a semana durante las 14 semanas de entrenamiento. Este trabajo de fuerza se combinó con trabajo de resistencia aeróbica realizado con cicloergómetro de pedalina (Magic, Spain) donde se trabajaba de forma continuada o fraccionada hasta alcanzar un total de 30 minutos realizados a una intensidad de 12-14 puntos de la escala de 20 puntos de Borg ${ }^{13}$.

Los ejercicios de fuerza se realizaron con banda elástica y tobilleras lastradas (Therabands TM, Adidas) y se llevaron a cabo los siguientes ejercicios: 1. Flexión-Extensión plantar de tobillo, 2. Flexión-Extensión de Cadera y Rodilla combinada: (Posición 1. Flexión de cadera. Posición 2. Extensión de rodilla. Posición 3. Flexión de rodilla. Posición 4. Extensión de cadera), 3. Abducción de cadera, 4. Aducción de cadera, mediante la compresión de un balón de goma-espuma, 5. Abdominales (mediante incorporaciones desde el sillón). Se progresó desde 1 serie hasta 3 series y desde 8-10 repeticiones por ejercicio hasta 10-12 repeticiones por ejercicio.

\section{Grado de cumplimiento:}

El número de sesiones totales de ejercicio fue de 40. Durante el proceso de intervención se analizó el grado de cumplimiento diario de cada uno de los sujetos, estableciendo 4 grupos en función del número de sesiones realizadas: grupo 1 (0-20 sesiones realizadas, cumplimiento $<50 \%$ ); grupo 2 (21-30 sesiones realizadas, cumplimiento 50-75\%); grupo 3 (31-36 sesiones realizadas, cumplimiento 75-90\%); grupo 4 (37-40 sesiones realizadas, cumplimiento 90-100\%).

\section{Análisis estadístico:}

La normalidad de la distribución de las variables se evaluó con la prueba de Shapiro-Wilk. Las variables con distribución normal analizadas se expresaron como Media \pm DE. Para comparar las variables analizadas entre los momentos $A-B$ y $B-C$ se utilizó el test paramétrico de muestras relacionadas. Para comparar las variables analizadas en el momento $B$ en función del grado de cumplimiento se utilizó el test paramétrico de muestras independientes t-student. Se consideró 
la $p \leq 0,05$ como valor significativo. Todos los análisis se realizaron mediante el programa estadístico SPSS (the Statitical Package for the Social Sciences Inc. Chicago, USA) versión 23.0.

\section{RESULTADOS}

\section{Características basales:}

Los pacientes participantes en el estudio fueron 29 varones con una edad media de $72,18 \pm 8,47$ años, peso de $73,77 \pm 11,22 \mathrm{~kg}$, talla de $167,80 \pm 6,50 \mathrm{~cm}$ e IMC de 26,19 $\pm 3,58 \mathrm{~m} / \mathrm{kg}^{2}$ (Tabla 1).

El rango de tiempo en HD de los pacientes es entre 3 y 8 años. Todos los sujetos estaban sometidos a 3 días de diálisis a la semana y una media de 3 horas y 45 minutos/día (Tabla 1).

Tabla 1. Descriptivo de las características antropométricas y clínicas de los pacientes con ERC sometidos a hemodiálisis en el momento $\mathrm{A}$.

\begin{tabular}{|c|c|c|}
\hline & $\begin{array}{c}\text { Media } \pm D E \\
(n=29)\end{array}$ & IC95\% \\
\hline Edad (años) & $72,18 \pm 8,47$ & {$[69,40-74,97]$} \\
\hline Peso $(\mathrm{kg})$ & $73,77 \pm 11,22$ & {$[69,58-77,96]$} \\
\hline Talla $(\mathrm{m})$ & $167,80 \pm 6,50$ & {$[165,37-170,23]$} \\
\hline $\mathrm{IMC}(\mathrm{kg} / \mathrm{m} 2)$ & $26,19 \pm 3,58$ & {$[24,85-27,52]$} \\
\hline Tratamiento en hemodiálisis (años) & $3,01 \pm 1,17$ & {$[2,68-3,35]$} \\
\hline Hemodiálisis (minutos/semana) & $667,05 \pm 44,62$ & {$[654,50-679,60]$} \\
\hline \multicolumn{3}{|l|}{ Enfermedad cronica } \\
\hline Hipertensión (no casos) & 15 & \\
\hline Depresión ( $n^{\circ}$ casos) & 1 & \\
\hline Diabetes ( $\mathrm{n}^{\circ}$ casos) & 9 & \\
\hline Infarto de miocardio ( $n^{\circ}$ casos) & 2 & \\
\hline Ictus ( $\mathrm{n}^{\circ}$ casos) & 1 & \\
\hline Otras ( $n^{\circ}$ casos) & 1 & \\
\hline \multicolumn{3}{|l|}{ Etiología de la ERC } \\
\hline Glomerulonephritis ( $\mathrm{n}^{\circ}$ casos) & 2 & \\
\hline Diabetes (nํㅡ casos) & 7 & \\
\hline Hipertensión (no casos) & 9 & \\
\hline Isquemia ( $n^{\circ}$ casos) & 1 & \\
\hline Enfermedad poliquística renal ( $n^{\circ}$ casos) & 1 & \\
\hline Nefropatía $\lg \mathrm{A}$ ( $\mathrm{n}^{\circ}$ casos) & 0 & \\
\hline Uso de analgésicos ( $\mathrm{n}^{\circ}$ casos) & 0 & \\
\hline Lupus eritematoso ( $\mathrm{n}^{\circ}$ casos) & 0 & \\
\hline Otros ( $\mathrm{n}^{\circ}$ casos $)$ & 9 & \\
\hline
\end{tabular}

DE: desviación estándar; ERC: enfermedad renal crónica; IC: Intervalo de confianza; kg: kilogramo; m: metros; n: número de sujetos. 


\section{Análisis del periodo sin intervención de ejercicio:}

Para el análisis de la condición física en el periodo A-B se bloqueó por sexo debido al reducido número de mujeres con ERC participantes en el estudio. De los 40 hombres seleccionados, 11 abandonaron el proyecto quedando una $\mathrm{N}$ total para la valoración de la condición física de 29 sujetos (Figura 1).

La fuerza de extremidades superiores en ambas manos descendió de forma significativa en el periodo de 14 semanas sin intervención (mano derecha: $28,36 \pm 6,66 \mathrm{~kg}$ vs $26,48 \pm 7,95 \mathrm{~kg}$ ( $p=0,017)$; mano izquierda: $25,85 \pm 7,41 \mathrm{vs}$ $24,43 \pm 7,99 \mathrm{~kg}(\mathrm{p}=0.041))$. Asimismo, en la fuerza-resistencia de extremidades inferiores valorada con los test funcionales STS30 y STS60 también descendieron de forma significativa el número de repeticiones en este mismo periodo $(11 \pm 3$ vs $10 \pm 3$ repeticiones $(p=0,010) ; 20 \pm 6$ vs $18 \pm 7$ repeticiones $(p=0,005)$, respectivamente). En el test STS10 se observó se un incremento significativo en el tiempo de ejecución $(30,89 \pm 8,79$ vs $35,47 \pm 13,56 \mathrm{seg}(\mathrm{p}=0,022)$. Por otro lado, el test indicativo de condición aeróbica (6MWT) no muestra cambios significativos, aunque disminuyó el número de metros recorridos en 6 minutos (Tabla 2).

Tabla 2. Resultados de condición física entre el momento $A$ y $B$ (sin intervención de ejercicio intradiálisis) y entre el momento $B$ y $C$ (con intervención de ejercicio intradiálisis).

\begin{tabular}{|c|c|c|c|c|c|c|c|c|}
\hline \multirow[t]{3}{*}{ Variable } & \multirow{3}{*}{$\mathbf{n}$} & \multicolumn{2}{|c|}{ De momento A a B } & \multirow{3}{*}{$\mathbf{p}$} & \multirow{3}{*}{$\mathbf{n}$} & \multicolumn{2}{|c|}{ De momento B a C } & \multirow{3}{*}{$\mathbf{p}$} \\
\hline & & A & B & & & B & C & \\
\hline & & Media $\pm D E$ & Media $\pm D E$ & & & Media $\pm D E$ & Media $\pm D E$ & \\
\hline \multicolumn{9}{|l|}{$\begin{array}{l}\text { Capacidad } \\
\text { aeróbica }\end{array}$} \\
\hline 6MWT (m) & 29 & $\begin{array}{c}323,71 \pm \\
126,57\end{array}$ & $\begin{array}{c}318,69 \pm \\
111,12\end{array}$ & 0,623 & 27 & $\begin{array}{c}322,54 \pm \\
106,98\end{array}$ & $\begin{array}{c}297,34 \pm \\
115,21\end{array}$ & 0,027 \\
\hline \multicolumn{9}{|l|}{$\begin{array}{l}\text { Fuerza de } \\
\text { extremidad } \\
\text { superior }\end{array}$} \\
\hline $\begin{array}{l}\text { Mano derecha } \\
(\mathrm{kg})\end{array}$ & 28 & $28,36 \pm 6,66$ & $26,48 \pm 7,95$ & 0,017 & 27 & $26,54 \pm 8,06$ & $26,76 \pm 8,81$ & 0,763 \\
\hline $\begin{array}{l}\text { Mano izquierda } \\
(\mathrm{kg})\end{array}$ & 28 & $25,85 \pm 7,41$ & $24,43 \pm 7,99$ & 0,041 & 27 & $24,54 \pm 8,29$ & $24,73 \pm 8,28$ & 0,803 \\
\hline \multicolumn{9}{|l|}{$\begin{array}{l}\text { Fuerza de } \\
\text { extremidades } \\
\text { inferiores }\end{array}$} \\
\hline $\begin{array}{l}\text { Chair Stand } \\
\text { Test } 30 \\
\text { (repeticiones) }\end{array}$ & 24 & $11 \pm 3$ & $10 \pm 3$ & 0,010 & 19 & $10 \pm 3$ & $11 \pm 3$ & 0,268 \\
\hline $\begin{array}{l}\text { Chair Stand } \\
\text { Test } 60 \\
\text { (repeticiones) }\end{array}$ & 24 & $20 \pm 6$ & $18 \pm 7$ & 0,005 & 19 & $19 \pm 7$ & $19 \pm 7$ & 0,509 \\
\hline $\begin{array}{l}\text { Sit to Stand to } \\
\text { Sit (s) }\end{array}$ & 24 & $30,9 \pm 8,8$ & $35,5 \pm 13,6$ & 0,022 & 19 & $33,0 \pm 11,3$ & $31,6 \pm 9,1$ & 0,320 \\
\hline
\end{tabular}

6MWT: 6 minutes walk test; DE: desviación estándar; kg: kilogramos; m: metros; n: número de sujetos; s: segundos 


\section{Análisis del periodo con intervención de ejercicio:}

Tras un periodo de 14 semanas de intervención con ejercicio físico, la fuerza de las extremidades superiores, medidas a través de dinamometría de manual y la fuerza-resistencia, medida por los test STS30, STS60 y STS10, no mostraron cambios estadísticamente significativos entre los momentos $\mathrm{B}$ y $\mathrm{C}$ en ninguna de las variables analizadas, frenándose el deterioro de la condición física durante el periodo de intervención con ejercicio físico (Tabla 2; Figura 2).

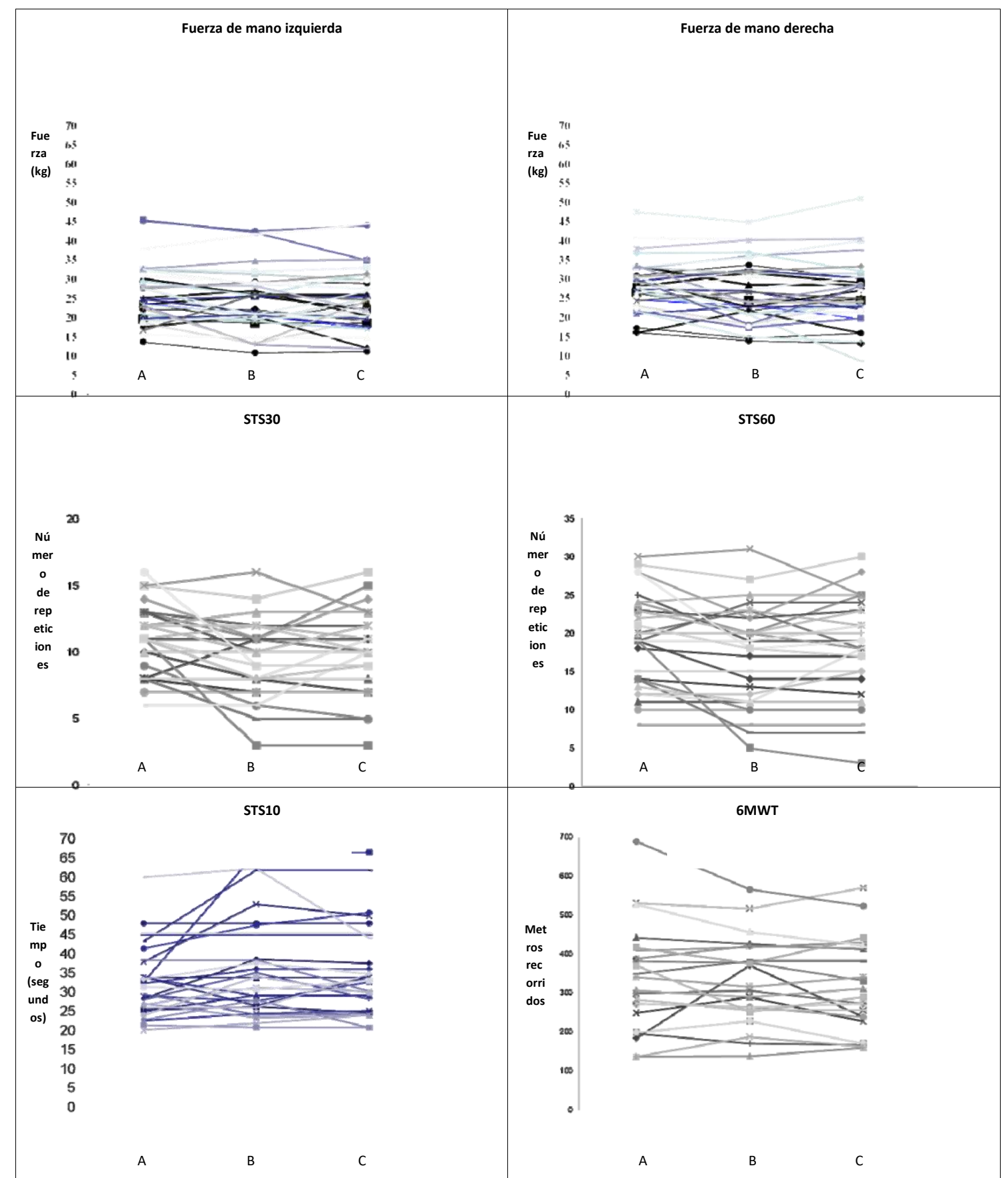

Figura 2. Flujo de pacientes para las variables de condición física desde los periodos A-B y $B-C$. 
En lo referente al test 6MWT, se observaron cambios significativos en la capacidad aeróbica disminuyendo los metros recorridos en 6 minutos (Tabla 2). Respecto al grado de cumplimiento del programa de ejercicio, se determinó que un $8,3 \%$ cumplieron menos del $50 \%$ de las sesiones de ejercicio, un $27,8 \%$ el $50-75 \%$, un $27,8 \%$ el $75-90 \%$, y un $36,1 \%$ realizaron el $90-100 \%$ de las sesiones de ejercicio.

Si estimamos que la dosis mínima efectiva debe estar por encima del $75 \%$ en el grado de cumplimiento, el 63,9\% del grupo participante consiguió alcanzar dicha dosis. Si analizamos las variables correspondientes a los datos antropométricos y de las variables de condición física en el momento $B$ antes de iniciar el programa de ejercicio en los pacientes con menos de un $75 \%$ y en los pacientes con más de $75 \%$ de cumplimiento no se encuentran diferencias significativas, es decir, ambos grupos según el grado de cumplimiento parten de las misma situación de condición física (Tabla 3).

Tabla 3. Biomarcadores y variables de condición física en el momento B según grado de cumplimiento del programa de intervención de ejercicio intradiálisis.

\begin{tabular}{|c|c|c|c|}
\hline & $\begin{array}{c}\text { Grado de } \\
\text { cumplimiento de } \\
\text { menos del } 75 \%\end{array}$ & $\begin{array}{l}\text { Grado de } \\
\text { cumplimiento de } \\
\text { más del } 75 \%\end{array}$ & $\mathrm{p}$ \\
\hline & $N=11$ & $\mathrm{~N}=18$ & \\
\hline Peso (kg) & $78,58 \pm 16,33$ & $76,03 \pm 12,22$ & 0,568 \\
\hline IMC $\left(\mathrm{kg} / \mathrm{m}^{2}\right)$ & $28,17 \pm 5,63$ & $26,52 \pm 4,00$ & 0,277 \\
\hline \multicolumn{4}{|l|}{ Variables aeróbicas } \\
\hline $6 \mathrm{MWT}(\mathrm{m})$ & $298,16 \pm 113,41$ & $329,88 \pm 110,88$ & 0,435 \\
\hline \multicolumn{4}{|l|}{$\begin{array}{l}\text { Variables de fuerza } \\
\text { de extremidad superior }\end{array}$} \\
\hline Mano derecha $(\mathrm{kg})$ & $25,14 \pm 7,51$ & $27,11 \pm 9,39$ & 0,435 \\
\hline Mano izquierda $(\mathrm{kg})$ & $21,93 \pm 6,64$ & $26,08 \pm 8,49$ & 0,075 \\
\hline \multicolumn{4}{|c|}{$\begin{array}{l}\text { Test funcionales de fuerza } \\
\text { de extremidades inferiores }\end{array}$} \\
\hline $\begin{array}{l}\text { Chair Stand Test } 30 \\
\text { (repeticiones) }\end{array}$ & $9 \pm 4$ & $11 \pm 2$ & 0,234 \\
\hline $\begin{array}{l}\text { Chair Stand Test } 60 \\
\text { (repeticiones) }\end{array}$ & $15 \pm 7$ & $20 \pm 6$ & 0,084 \\
\hline Sit to Stand to Sit10 (s) & $42,23 \pm 16,11$ & $31,86 \pm 10,91$ & 0,080 \\
\hline
\end{tabular}

6MWT: 6 minutes walk test; DE: Desviación Estándar; kg: kilogramos; m: metros; n: número de sujetos; s: segundos

Atendiendo al grado de cumplimiento del programa de ejercicio, los resultados muestran que aquellos pacientes con alto grado de cumplimiento no disminuyeron su condición cardiorrespiratoria respecto a aquellos con bajo grado de cumplimiento del programa (Tabla 4 y Figura 3). Para las variables de fuerza de 
extremidades superiores e inferiores no se encontraron diferencias significativas entre ambos grupos de cumplimiento (Tabla 4 y Figura 3), pero sí se observó una tendencia mayor a frenar la pérdida para aquellos pacientes con un mayor cumplimiento al programa (Figura 3).

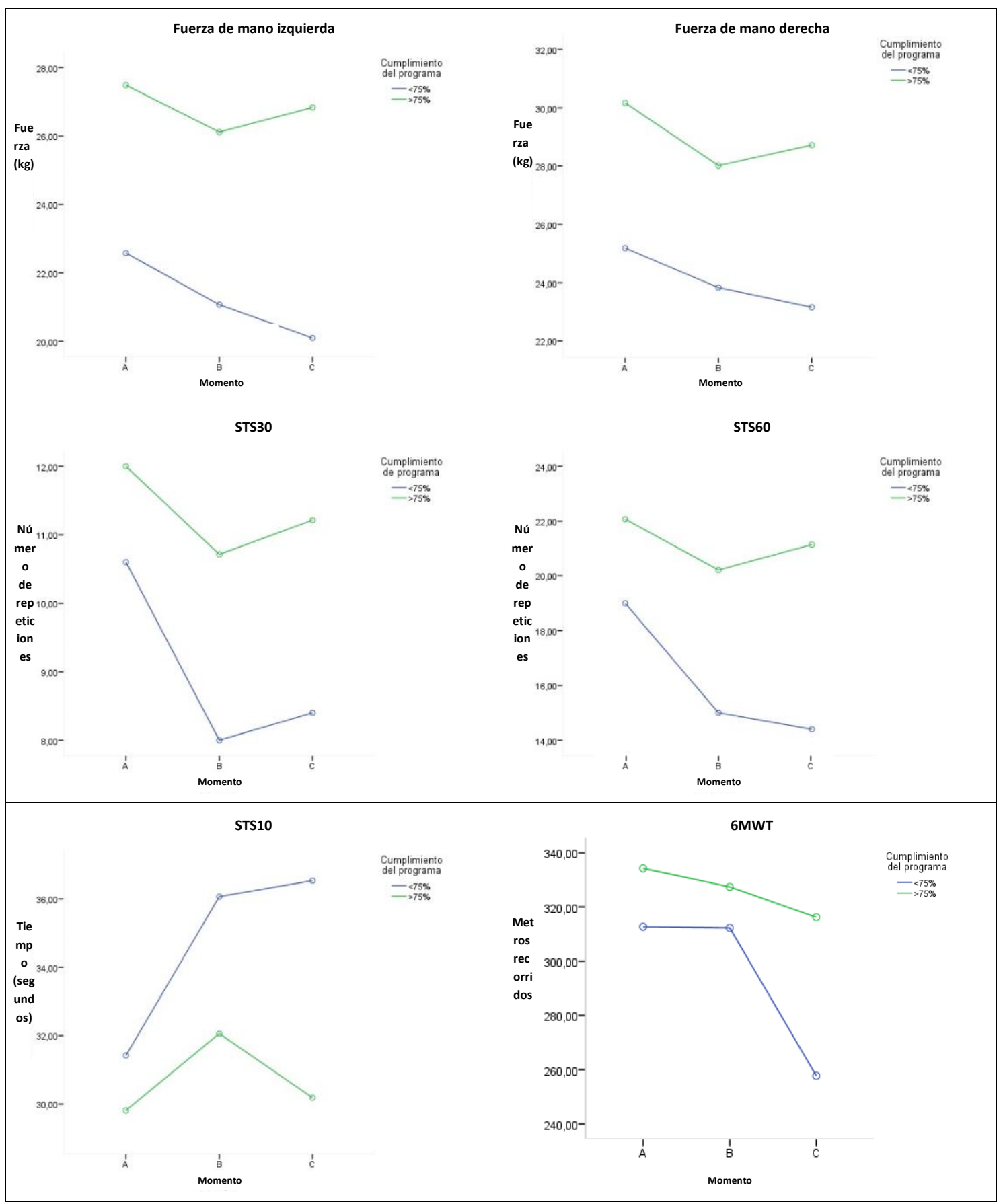

Figura 3. Condición física en momentos $\mathrm{A}$, B y C según el grado de cumplimiento del programa de ejercicio. 
Tabla 4. Resultados de condición física según grado de cumplimiento del programa de ejercicio intradiálisis.

\begin{tabular}{|c|c|c|c|c|c|c|c|c|c|}
\hline \multirow[t]{3}{*}{ Variable } & \multirow{3}{*}{$\begin{array}{l}\text { Grado de } \\
\text { cumplimiento } \\
\text { de dosis de } \\
\text { ejercicio }\end{array}$} & \multicolumn{4}{|c|}{ De momento A a B } & \multicolumn{4}{|c|}{ De momento B a C } \\
\hline & & $\mathrm{n}$ & A & B & $\mathrm{p}$ & $\mathrm{n}$ & B & C & $\mathrm{p}$ \\
\hline & & & Media $\pm D E$ & Media $\pm D E$ & & & Media $\pm D E$ & Media $\pm D E$ & \\
\hline \multicolumn{10}{|l|}{$\begin{array}{l}\text { Capacidad } \\
\text { aeróbica }\end{array}$} \\
\hline \multirow[t]{2}{*}{ 6MWT (m) } & $<75 \%$ & 11 & $303,29 \pm 108,89$ & $298,16 \pm 113,41$ & 0,754 & 10 & $312,32 \pm 98,02$ & $257,73 \pm 98,69$ & 0,056 \\
\hline & $\geq 75 \%$ & 18 & $334,85 \pm 136,37$ & $329,88 \pm 110,88$ & 0,711 & 17 & $327,40 \pm 112,99$ & $316,20 \pm 119,87$ & 0,266 \\
\hline \multicolumn{10}{|l|}{$\begin{array}{l}\text { Variables de fuerza } \\
\text { de extremidad } \\
\text { superior }\end{array}$} \\
\hline \multirow[t]{2}{*}{ Mano derecha (kg) } & $<75 \%$ & 10 & $25,30 \pm 5,87$ & $23,88 \pm 6,35$ & 0,180 & 10 & $23,83 \pm 6,63$ & $23,16 \pm 6,02$ & 0,525 \\
\hline & $\geq 75 \%$ & 18 & $30,17 \pm 6,54$ & $28,02 \pm 8,52$ & 0,052 & 17 & $28,02 \pm 8,52$ & $28,72 \pm 9,56$ & 0,469 \\
\hline \multirow[t]{2}{*}{ Mano izquierda (kg) } & $<75 \%$ & 10 & $22,95 \pm 5,40$ & $21,26 \pm 6,03$ & 0,054 & 10 & $21,07 \pm 6,41$ & $20,10 \pm 6,28$ & 0,483 \\
\hline & $\geq 75 \%$ & 18 & $27,36 \pm 7,96$ & $26,08 \pm 8,49$ & 0,189 & 17 & $26,11 \pm 8,69$ & $26,83 \pm 8,33$ & 0,443 \\
\hline \multicolumn{10}{|l|}{$\begin{array}{l}\text { Test funcionales } \\
\text { de fuerza de } \\
\text { extremidades } \\
\text { inferiores }\end{array}$} \\
\hline \multirow{2}{*}{$\begin{array}{l}\text { Chair Stand Test } 30 \\
\text { (repeticiones) }\end{array}$} & $<75 \%$ & 9 & $10 \pm 2$ & $8 \pm 3$ & 0,112 & 5 & $8 \pm 3$ & $8 \pm 4$ & 0,688 \\
\hline & $\geq 75 \%$ & 15 & $12 \pm 3$ & $11 \pm 3$ & 0,054 & 14 & $11 \pm 3$ & $11 \pm 3$ & 0,314 \\
\hline \multirow{2}{*}{$\begin{array}{l}\text { Chair Stand Test } 60 \\
\text { (repeticiones) }\end{array}$} & $<75 \%$ & 9 & $18 \pm 5$ & $14 \pm 6$ & 0,052 & 5 & $15 \pm 7$ & $14 \pm 8$ & 0,732 \\
\hline & $\geq 75 \%$ & 15 & $22 \pm 6$ & $20 \pm 6$ & 0,058 & 14 & $20 \pm 6$ & $21 \pm 5$ & 0,320 \\
\hline \multirow[t]{2}{*}{ Sit to Stand to Sit (s) } & $<75 \%$ & 8 & $32,4 \pm 6,2$ & $42,2 \pm 16,1$ & 0,064 & 4 & $36,1 \pm 12,7$ & $36,5 \pm 10,4$ & 0,857 \\
\hline & $\geq 75 \%$ & 15 & $30,1 \pm 10,0$ & $31,9 \pm 10,9$ & 0,151 & 14 & $32,1 \pm 11,3$ & $30,2 \pm 8,6$ & 0,255 \\
\hline
\end{tabular}

6MWT: 6 minutes walk test; DE: desviación estándar; kg: kilogramo; m: metro; n: número de sujetos; s: segundos

\section{DISCUSIÓN}

Los resultados más relevantes de este estudio nos informan de que el programa de ejercicio intradiálisis ayudó a frenar la pérdida de fuerza que origina la evolución y progresión de la ERC.

Durante el periodo sin intervención de ejercicio, se originó una pérdida significativa de la fuerza en extremidades superiores e inferiores afectando a la dinamometría manual y a los tests funcionales STS60, STS30 y STS10. Asimismo, se vieron afectados los resultados del test 6MWT disminuyendo el número de metros recorridos en el periodo en que no realizaron ejercicio intradiálisis, lo que nos indica un descenso de la capacidad cardiorrespiratoria. Los trabajos de Song y Sohng ${ }^{14}$ y Chen et al, ${ }^{15}$ corroboran estos resultados observándose como 
en los sujetos del grupo control no sometidos a ejercicio, se produce un deterioro de la condición física. Así, Song y Sohng ${ }^{14}$ en su estudio con entrenamiento de fuerza progresivo durante 12 semanas, encuentran que el grupo que realizó ejercicio aumentó de manera significativa la fuerza muscular en muslo izquierdo en 4,3 kg, mientras que el grupo control perdió 1,4 kg en la misma extremidad. Chen et $\mathrm{al}^{15}$ tras 48 sesiones de ejercicio a baja intensidad, encuentra un descenso en el componente físico del cuestionario SF-36 y pérdida de fuerza en su grupo control en contraposición a la mejora del grupo que sí hizo ejercicio físico. Los descensos en la condición física pueden repercutir en el pronóstico y supervivencia de la enfermedad crónica tal y como remarcan los trabajos de Myers et $\mathrm{al}^{16}$. En ellos se muestra que la tolerancia al ejercicio es un predictor fuertemente relacionado con la supervivencia en sujetos con patologías crónicas, más incluso que variables clínicas tales como la HTA, el hábito de fumar o la DM. Por su parte, Sietsema et $\mathrm{al}^{17}$ afirman que la tolerancia al ejercicio constituye un predictor de supervivencia en sujetos con enfermedad renal terminal. En estos estudios la variable utilizada fue el consumo de oxígeno pico para medir la capacidad cardiorrespiratoria, mientras que en nuestro estudio utilizamos un parámetro indirecto que se relaciona con dicho consumo de oxígeno y que fue el test 6MWT, variable que quizás no nos ha permitido poder evaluar con precisión la condición aeróbica en nuestra muestra. Cuando el grupo de pacientes fue sometido a ejercicio no consiguió mejoras significativas de la fuerza ni de la condición aeróbica pero sí consiguió frenar la pérdida, lo que puede considerarse de gran relevancia clínica en una enfermedad tan debilitante, tal y como concluyen los trabajos de Song y Sohng (14) o Cheema et al18. Los resultados obtenidos en nuestro estudio en el test 6MWT coinciden con los observados por el Grupo Canadiense de Estudio de la Eritropoyetina ${ }^{19}$.

La fuerza muscular de extremidades inferiores, medidas a través del test STS10, refleja un mantenimiento del tiempo empleado en realizar 10 sentadillas, remarcando el mantenimiento de la fuerza de extremidades inferiores. El índice de resistencia muscular de extremidades inferiores, analizado por los tests funcionales STS30 y STS60, se mantiene o aumenta de forma no significativa el número de repeticiones realizadas en 30 o 60 segundos. La fuerza máxima de prensión manual se mantuvo en el tiempo cuando se realizó un programa de ejercicio, siendo muy importante dada la relevancia clínica que supone este índice relacionado con la supervivencia del paciente con ERC, tal y como remarca el estudio de Wang et $\mathrm{al}^{20}$. El trabajo llevado a cabo por Singh ${ }^{8}$ recalca que la fuerza de manos es un predictor fundamental de muertes por eventos cardiovasculares, que constituyen a su vez una de las principales causas de muerte de este colectivo. Además, recientemente se ha propuesto que la valoración de la fuerza muscular es un mejor predictor de comorbilidades y consecuencias de la ERC que la evaluación de la masa muscular, lo que abriría un nuevo screening para la evaluación y oportunidades terapéuticas en sujetos enfermos de $\mathrm{ERC}^{7}$.

Los resultados encontrados difieren de otros estudios en los que encuentran cambios significativos de la fuerza y la condición aeróbica en aquellos pacientes sometidos a ejercicio intradiálisis ${ }^{18,21}$. Tal vez un programa realizado a mayor intensidad y/o más largo en el tiempo permite obtener mejoras más significativas. 
Greenwood et $\mathrm{al}^{21}$, tras una intervención de un año con ejercicio aeróbico en las sesiones de hemodiálisis ( 3 veces por semana), consiguieron, entre otras, mejoras significativas del volumen de oxígeno pico. Cheema et al ${ }^{18}$, trabajando con entrenamiento de fuerza progresivo a intensidades altas llegaron a obtener mejoras significativas en la morfología muscular, fuerza y calidad de vida. En la búsqueda de un protocolo de ejercicio ideal para este colectivo, tanto la duración como la intensidad serían dos puntos clave para conocer la dosis en la que las mejoras pueden ayudar a conseguir una mejor condición física, que les permitiese alcanzar una mejor evolución de la enfermedad y tal vez una mayor supervivencia. Cuando diferenciamos los sujetos teniendo en cuenta si el grado de cumplimiento es superior o inferior al $75 \%$ del programa, existe una tendencia a mantener más elevados los niveles de condición física en aquellos pacientes que cumplieron al menos el $75 \%$ del programa de ejercicio, lo que apoya la necesidad de alcanzar una dosis suficiente de ejercicio para obtener unas mejoras en la condición física.

\section{CONCLUSIÓN}

Los resultados obtenidos en el programa de ejercicio intradiálisis demuestran la importancia del ejercicio para frenar el deterioro de la condición física ocasionada por la ERC. Futuros estudios deberán encontrar la carga adecuada para conseguir mejoras significativas e incluir un tiempo de intervención largo.

\section{REFERENCIAS BIBLIOGRÁFICAS}

1. Jha V, García-Cargía G, Iseki K, Li Z, Naicker S, Plattner B, et al. Chronic kidney disease: global dimension and perspectives. Lancet. 2013 Jul 20;382(9888):260-72.

2. Kouidi E, Albani M, Natsis K, Megalopoulos A, Gigis P, Guiba-Tziampiri O, et al. The effects of exercise training on muscle atrophy in hemodialysis patients. Nephrol Dial Transplant 1998; 13: 685-699.

3. Caso G, Garlick P: Control of muscle protein kinetics by acid-base balance. Curr Opin Clin Nutr Metab Care 2005; 8: 73-76.

4. Raj D, Zager P, Shah V, Dominic E, Adeniyi O, Blandon P, et al. Protein turnover and amino acid transport kinetics in end-stage renal disease. Am J Physiol Endocrinol Metab 2004; 286: E136-E143.

5. Avesani CM, Trolonge S, Deleaval P, Baria F, Mafra D, Faxen-Irving G, et al. Physical activity and energy expenditure in haemodialysis patients: an international survey. Nephrol Dial Transplant Off Publ Eur Dial Transplant Assoc Eur Ren Assoc. 2012;27:2430-4.

6. Segura-Ortí, E. Ejercicio en pacientes en hemodiálisis: revisión sistemática de la literatura. Nefrología (Madrid), 2010; 30(2): 236-246.

7. Stenvinkel P, Carrero JJ, von Walden F, Ikizler TA, Nader GA. Muscle wasting in end-stage renal disease promulgates premature death: established, emerging and potential novel treatment strategies. Nephrol Dial Transplant. 2015 Apr 24.

8. Singh MA: Exercise comes of age: Rationale and Recommendations for a geriatric exercise prescription. J Gerontol A Biol Sci Med Sci 2002; 57A: M262-M282. 
9. Johansen K, Painter P, Sakkas G, Gordon G, Gordon P, Doyle J, Shubert T. Effects of resistance exercise training and nandrolone decanoate on body composition and muscle function among patients who receive hemodialysis: A randomized controlled trial. J Am Soc Nephrol 2006; 17: 2307- 2314.

10. Rikli, R. \& Jones, C.J. Senior fitness test manual. United States: Human Kinetics; 2001.

11. Oja P; Tuxworth B. Eurofit para adultos. Test Europeo de aptitud física. Evaluación de la aptitud física en relación con la salud. Deportes CS de, editor. Madrid: Ministerio de Educación y Ciencia; 1998

12. Segura-Ortí, E., Martínez-Olmos, FJ. Test-retest reliability and minimal detectable change scores for sit-to-stand-to-sit tests, the six-minute walk test, the one-leg heelrise test, and handgrip strength in people undergoing hemodialysis. Phys Ther. 2011 Aug;91(8):1244-52.

13. Burkhalter N. Evaluación de la escala Borg de esfuerzo percibido aplicada a la rehabilitación cardiaca. Rev Lat Am Enfermagem [Internet]. 1996;4(3):65-73.

14. Song WJ, Sohng KY. Effects of progressive resistance training on body composition, physical fitness and quality of life of patients on hemodialysis. J Korean Acad Nurs. 2012 Dec;42(7):947-56.

15. Chen JL, Godfrey S, Ng TT, Moorthi R, Liangos O, Ruthazer R, et al. Effect of intradialytic, low-intensity strength training on functional capacity in adult haemodialysis patients: a randomized pilot trial. Nephrol Dial Transplant. 2010 Jun;25(6):1936-43.

16. Myers J, Prakash M, Froelicher V, Do D, Partington S, Atwood E. Exercise Capacity and Mortality among Men Referred for Exercise Testing. N Engl J Med 2002; 346:793-801

17. Sietsema K, Amato A, Adler SG, Brass EP. Exercise capacity as a predictor of survival among ambulatory patients with end-stage renal disease. Kidney International. 2004 (65): 719-724.

18. Cheema B, Abas H, Smith B, O'Sullivan A, Chan M, Patwardhan A, et al. Randomized controlled trial of intradialytic resistance training to target muscle wasting in ESRD: the Progressive Exercise for Anabolism in Kidney Disease (PEAK) study. Kidney Dis. 2007 Oct;50(4):574-84

19. Canadian Erythropoietin Study Group. Association between recombinant human erythropoietin and quality of life and exercise capacity of patients receiving haemodialysis.. BMJ. 1990 3;300(6724):573-8.

20. Wang AY, Sea MM, Ho ZS, Lui SJ, Li PK, Woo J. Evaluation of handgrip strength as a nutritional marker and prognostic indicator in peritoneal dialysis patients. Am J Clin Nutr. 2005 Jan;81(1):79-86

21. Greenwood SA, Koufaki P, Mercer TH, MacLaughlin HL, Rush R, Lindup H, et al. Effect of exercise training on estimated GFR, vascular health, and cardiorespiratory fitness in patients with CKD: a pilot randomized controlled trial. Am J Kidney Dis. 2015 Mar,65(3):425-34.

Referencias totales citadas: 21

Referencias citadas correspondientes a la Rev lb CC Act Fis Dep: 0 